\title{
Frozen Phase in Situ Multi-Modal Microscopy of Liquid Metal Eutectics
}

\author{
Austin J. Akey ${ }^{1}$ and David C. Bell ${ }^{1,2}$ \\ 1. Center for Nanoscale Systems, Harvard University, Cambridge, MA. \\ 2. Harvard John A. Paulson School of Engineering and Applied Sciences, Harvard University, \\ Cambridge, MA.
}

In recent years, there has been a growing interest in the use in-situ Transmission Electron Microscopy (TEM) methods to directly observe structural changes in functional material during operation. State-ofthe-art in-situ electron microscopy techniques can monitor morphological and crystallographic changes in real time with high spatial and temporal resolution [1-3]. However, compositional information can at present only be obtained using slow techniques that rely on low signal-to-noise measurements such as EDS or EELS; these measurements have spatial resolutions on the order of many nm, significantly coarser than the imaging to which they are correlated. To overcome these limitations, we develop a new multi-modal microscopy method which combines the spatiotemporal structural imaging of in-situ TEM with the spatiochemical compositional analysis of Atom Probe Tomography (APT).

To demonstrate this method, we present correlated in-situ spatiostructural and ex-situ spatiochemical measurements of a Liquid Metal Eutectic (LME) system in contact with a solid metal substrate. The use of LMEs as heat transfer fluids, coolants, and radiation shielding materials is the subject of intense investigation for concentrated solar power installations and generation IV nuclear fission power systems, with the most heavily-studied LME systems being $\mathrm{NaK}$ and $\mathrm{PbBi}$ [4]. Liquid $\mathrm{NaK}$ has numerous safety problems due to its extreme reactivity on exposure to ambient conditions, so $\mathrm{PbBi}$ is generally preferred; however, this LME is known to be highly corrosive to structural metals, with significant research underway into the mechanism of corrosion, the timeline of the degradation process, and its mitigation [5]. Direct analysis of the atomic-scale structural and chemical changes in these material systems has the potential to greatly accelerate progress towards safe, cost-effective application of liquid metal heattransfer systems.

In the present work, we use in-situ TEM with heating to observe first the melting of an LME alloy deposited onto a metallic substrate, and then the structural changes induced in the substrate at elevated temperatures by contact with the LME, including penetration of grain boundaries and modification of the substrate surface morphology. By rapidly reducing the temperature of the system when structural changes are observed, it is possible to "freeze" those changes into the material, allowing the same specimen used for TEM to be converted into an APT specimen with minimally-disruptive additional processing. The structural changes observed in the TEM specimen can then be directly analyzed using APT's atomic-scale compositional sensitivity, allowing direct, one-to-one correlation of the two techniques. We present the results of model experiments performed with $\mathrm{PbBi}$ on structural metal alloy substrates, and demonstrate correlative measurement of in-situ observation of structural modification through exposure to LME and atomic-scale composition changes at specific sites within the substrate. We demonstrate the potential for real-time observation of structural evolution in materials with the ability to perform ultra-high-resolution chemical analysis at an arbitrary point in the process, opening the doors to a wide range of time-resolved experiments in materials science and chemistry [6]. 
References:

[1] F. Tao et al, Chemical Communications 50 (2014), p. 12417.

[2] P.L. Gai and E.D. Boyes, Electron Microscopy and Analysis Group Conference IOPPublishing 522 (2013), p. 012002.

[3] H. Matsumoto et al, Microscopy and Analysis (2013).

[4] A. Heinzel et al, Energy Technology 5 (2017), p. 1026.

[5] R. Ballinger and J. Lim, Nuclear Technology 147(3) (2004), p. 418.

[6] This work was performed at the Center for Nanoscale Systems (CNS), a member of the National Nanotechnology Coordinated Infrastructure Network (NNCI), which is supported by the National Science Foundation under NSF award no. 1541959.

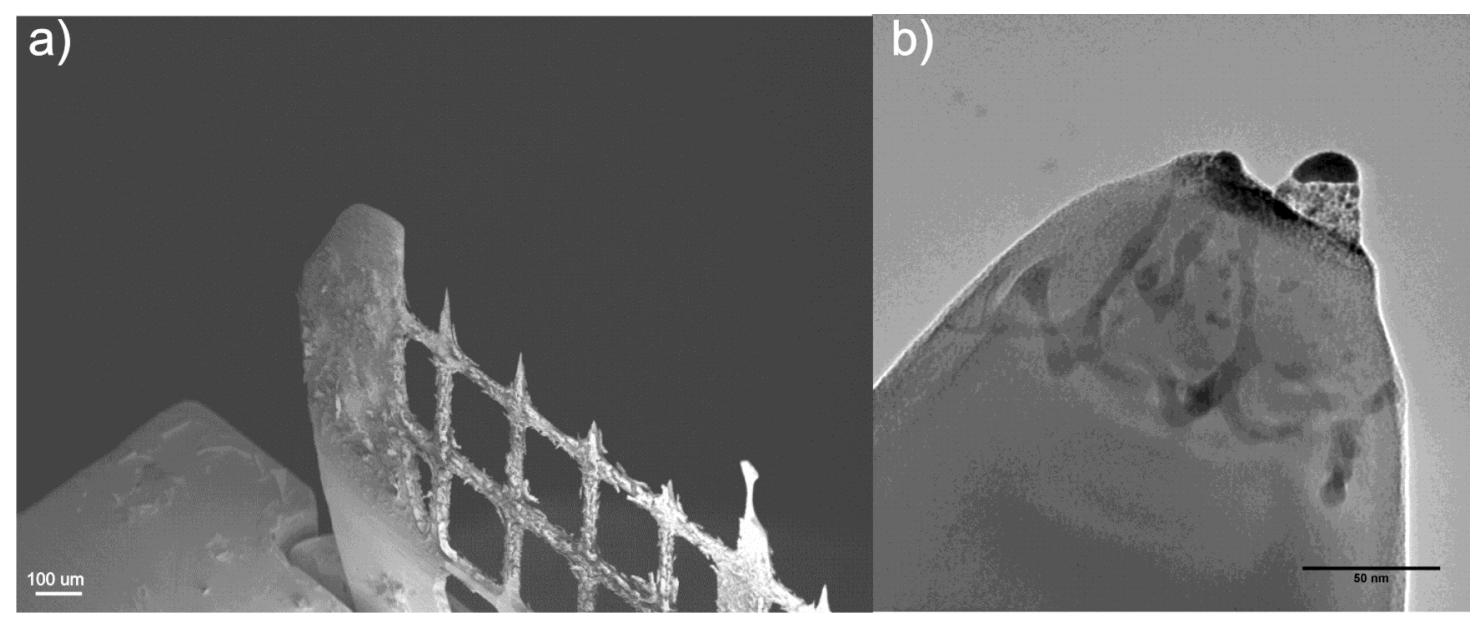

Figure 1. Correlative in-situ TEM/APT specimen for multimodal microscopy. a) SEM image of TEM half-grid prepared by electrochemical etching from conventional metal substrate b) APT tip formed from material following rapid heating and quenching, with structural and compositional features intact allowing direct TEM-APT correlation on individual specimens. 